\title{
HERA STATUS AND UPGRADE PLANS
}

\author{
F. Willeke, Deutsches Elektronen-Synchrotron, Notkestr.85, 22603 Hamburg, Germany
}

\section{Abstract}

The HERA electron-proton collider at Hamburg Germany, designed for collisions of $820 \mathrm{GeV}$ protons with $30 \mathrm{GeV}$ electrons is now in its 6th year of operation. It delivers luminosity for the colliding beam detectors H1 and ZEUS and longitudinally polarized positrons for the internal target experiment HERMES. HERA is operated close to the design luminosity with peak values of $1.2 \times 10^{31} \mathrm{~cm}^{-2} \mathrm{sec}-1$. While the beam currents still fall short of expectations, the luminosity was increased by focusing the beams very tightly at the interaction points at the expense of using up most of the margins on aperture, chromaticity and beam-beam tuneshift. In 1996, an integrated luminosity of $\int \mathcal{L} d t=17.2 p b^{-1}$ has been delivered. Despite the strong beam-beam interactions, the degree of longitudinal polarization of the positron beam is, with (50-60)\%, larger than expected.

Despite the good results of HERA, more luminosity is needed for the physics program in the next decade. Preparations are underway to increase the luminosity by rebuilding the low- $\beta$ insertions. With unconventional new types of magnets, it seems to be possible to increase the luminosity substantially. The expected value is $\mathcal{L}_{\text {peak }}=$ $7.5 \times 10^{31} \mathrm{~cm}^{-2} \mathrm{sec}^{-1}$ which is five times larger than the design value.

\section{INTRODUCTION}

The HERA collider[1] for $820 \mathrm{GeV}$ protons (p-beam) and $30 \mathrm{GeV}$ electrons or positrons (e-beam) in Hamburg is the only high-energy accelerator in which two completely different species of particles are brought into collision. The double ring collider has a length of $6.3 \mathrm{~km}$. The proton ring has a superconducting magnet structure with $4.6 \mathrm{~T}$ magnets. The electron ring has conventional magnets but needs, besides 84 room temperature rf cavities, a superconducting cavity system to reach at the current top-energy of $27.5 \mathrm{GeV}$. HERA has two interaction regions for the colliding beam experiments $\mathrm{H} 1$ and ZEUS. In addition it hosts two fixed target experiments, the gas target experiment HERMES which needs a longitudinally polarized electron or positron beam and the new HERA-B experiment which is going to analyze the interactions of halo protons with a wire target.

The collider has been providing luminosity for more than five years now. It is operated close to the design values for luminosity and spin polarization. The recent incorporation of the HERA-B target operation in HERA operations was a success. At this point four different experiments with very different demands on the accelerator performance are running simultaneously without much detrimental interference.
The results from the HERA experiments open up a rich future physics programme. It is therefore highly desirable to produce luminosity in excess of what the present design can deliver. Therefore, plans are underway to rebuild the collider interaction regions (IR) to allow stronger focusing of both beams.

\section{HERA LUMINOSITY OPERATION}

HERA luminosity operation has made steady progress. The machine is now operated close to design conditions. Since 1994 HERA has been operated with $27.5 \mathrm{GeV}$ positrons which collide with $820 \mathrm{GeV}$ protons. At this point the peak luminosity is $1.2 \times 10^{31} \mathrm{~cm}^{-2} \mathrm{sec}^{-1}$, the number of protons per bunch is up to $6 \times 10^{10}$, the total beam current of the positrons reaches values of up to $44 \mathrm{~mA}$. The normalized emittance of the p-beam is in the order of $4 \mu \mathrm{m}$ in collisions. The beam-beam tune shift of the positrons of $\Delta \nu_{y e} \simeq 0.03$ exceeds already the design value by a factor of 1.5. While the beam currents quoted above are still below expectations, the matched beam cross section at the interaction point is reduced by more than a factor compared to the design value. Thus high luminosity in HERA is achieved by cutting into the margins of aperture, chromaticity of the protons and of beam-beam tune-shift of the leptons.

Great progress has been made in the last two years on overall reliability. In 1997, HERA is delivering luminosity to the experiments for nearly $50 \%$ of the scheduled time. The minimum time needed to re-inject, to accelerate and to tune the two beams amounts to about $20 \%$ of the scheduled time. The remaining $30 \%$ are still needed at this point for optimizations or recovery from failures. The recent progress is the result of preventive maintenance on the more than thousand magnet power supplies[3] and regular reconditioning of the superconducting rf cavities. Furthermore a large effort is underway to store accelerator data in circular buffers to ease trouble shooting on the numerous components[2].

In 1996, HERA delivered an integrated luminosity of $17.2 p b^{-1}$ to the colliding beam experiments $\mathrm{H} 1$ and ZEUS. At present, luminosity is produced at a monthly rate of $4.5 p b^{-1}$. The continuous improvement of HERA operations is reflected in Fig. 1 which shows the integrated luminosity versus the running time for the years 1992-1997.

Since 1995, the HERMES experiment investigates the reactions of high energy longitudinally spin-polarized positrons with a polarized gas target. HERA has a pair of spin rotator magnets installed around the experiment, which rotate the vertically oriented spin into the longitudinal direction and back into the vertical direction. The polarization is usually between $50 \%$ and $60 \%$ in routine 


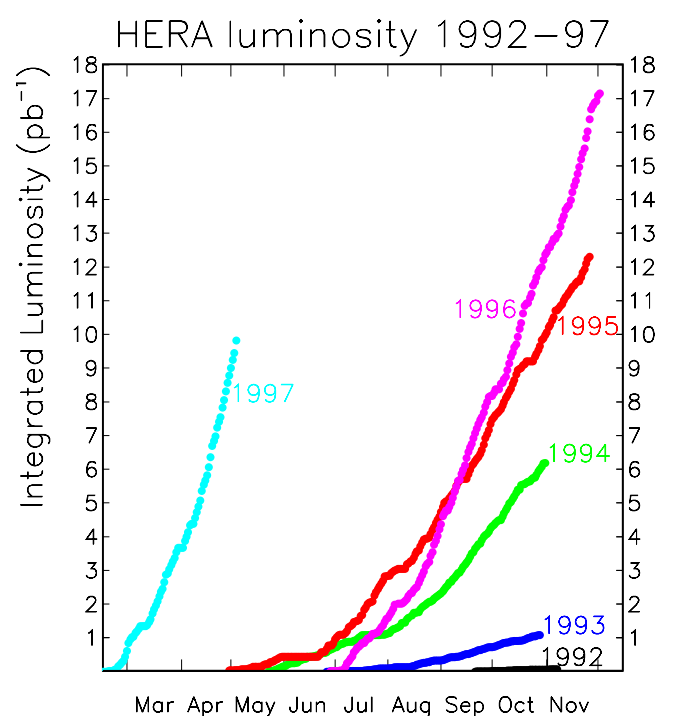

Figure 1: Accumulation of HERA Luminosities 1992-1997

operation. Peak values of up to $70 \%$ have been observed. The polarization is remarkably stable. Major tuning with harmonic orbit bumps[7] is performed only once or twice per year. High polarization can then be maintained by occasional orbit corrections. There is some indication, that high polarization is more difficult to obtain with strong beam-beam interactions. In 1996, there was some correlation was observed between beam-beam tune-shift of the positron beam and spin polarization. The tuneshift reached values of $\Delta \nu=0.035$ and sometimes the polarization approached a level of $50 \%$ only after many hours of slow growth (The polarization time in HERA at $27.5 \mathrm{GeV}$ is less than 37 minutes). More careful control of the tunes inside a very narrow window reduced this problem. Recently the vertical $\beta$ function of the positron beam has been reduced at the interaction point (IP). Since then no correlation between proton beam intensity and positron spin polarization has been observed.

\section{HERA ELECTRON OPERATION}

A very unpleasant surprise was that the lifetime of intense electron beams in HERA is not determined by bremsstrahlung at desorbed gas molecules as expected but by effects which have been explained by dust trapped inside the beam. The present understanding is that dust particles are emitted from the chamber of the integrated ion getter pumps by an unknown mechanism. This effect makes the operation with electrons virtually impossible. Since 1994 HERA has been operated with positrons and there are no anomalies in the beam lifetime. The dust or micro-particles hypothesis has been supported by many experiments $[5,4,6]$. All efforts to overcome this problem have failed so far. It has been attempted to lower the voltage of the integrated pumps. This was not successful. Turning off the pumps completely seems to suppress the effect. This leads however to unsatisfactory lifetime due to increased pressure of desorbed gas. It has been attempted to drive out trapped particles by kicking the beam. This provides relief only for a period of several minutes. Then the lifetime breaks down again. BPM monitors have been used as clearing electrodes. But this did not solve the problem. The last attempt to resolve the problem, without major changes in the pumping system, was to equip all integrated pumps with pre-resistors of $5 M \Omega$. This did not improve the situation.

In the winter shutdown period 1995-1996, an $\simeq 120 \mathrm{~m}$ long section of HERA has been equipped with passive nonevaporating getter pumps (NEG[8]). These pumps have been operated without any problems in the 1996 and 1997 luminosity operation. Pumping speed and required generation are within expectations. Experiments with electron beams indicate, that in this vacuum section, the local beam losses due to bremsstrahlung events have been drastically reduced after the NEG pumps have been installed. Electron beam experiments with all getter pumps turned off have been performed in December 1996. They seem also to indicate that the lifetime degradation is removed if there are no getter pumps present and electrons behave the same as positrons. Therefore, the pumping system in the HERA ering is planned to be replaced by NEG pumps in the winter shut-down 1997/1998.

\section{HERA LUMINOSITY LIMITATIONS}

The Luminosity of HERA is best written as

$$
\mathcal{L}=\frac{N_{p} I_{e}}{4 \pi e \sigma_{x} \sigma_{y}}
$$

( $N_{p}$ is the number of protons per bunch, $I_{e}$ is the total beam current of the lepton beam, $e$ is the elementary charge, $\sigma_{x, y}$ are the rms beam sizes of the p-beam or e-beam with $\sigma_{x, y}^{p}=\sigma_{x y}^{e}$ ).

With a bunch spacing of 96 ns HERA luminosity is limited by the total lepton beam current and the beam current is limited by the available rf power. At present, the rf power is sufficient to store $45 \mathrm{~mA}$ reliably at $27.5 \mathrm{GeV}$, the lowest possible energy which allows longitudinal spin polarization. An eighth rf station is under construction to raise this limit above $50 \mathrm{~mA}$. The total available rf power will be $12 \mathrm{MW}$ which will allow the design beam current of $58 \mathrm{~mA}$ to be stored at $27.5 \mathrm{GeV}$.

The bunch intensity of the protons is limited by space charge effects at injection into the booster. The incoherent space charge tuneshift at $50 \mathrm{MeV}$ injection into the synchrotron DESYIII is $\Delta Q_{\text {incoh }}=0.4$ (see also[10]), which corresponds to about $1.2 \times 10^{11}$ protons per bunch at extraction into the PETRA injector ring. Tight aperture in the beam lines and dynamic aperture limitations in the whole proton acceleration chain up to $70 \mathrm{GeV}$ lead to beam losses so that only $6 \times 10^{10}$ protons per bunch can be reached at present in HERA luminosity operation.

The beam cross sections of positrons and protons need to be matched to keep nonlinear effects of the beam-beam 
interaction to a minimum. The beam width and height of the protons are given by the product of emittance and $\beta$ functions.

The limit on p-beam emittance, $\varepsilon$, is closely related to the p-beam intensity by the maximum brightness $N_{p} / \varepsilon_{N}$ achieved in DESYIII. With the present intensity the normalized transverse emittance in HERA is $\varepsilon_{N}=(4-$ 5) $\mu \mathrm{m}$. Electron-proton collisions require short proton bunch lengths. This means that the longitudinal emittance budget is also tight and the bunch intensity cannot be further increased by $\mathrm{rf}$ manipulations.

For the e-beam, the low- $\beta$ quadrupoles are placed much closer to the IP than the p-beam quadrupoles. Despite the small proton emittance of $5 \mathrm{~nm}$ at $820 \mathrm{GeV}$ the cross section is limited by the $\beta$-function of the protons. There are in the present lattice no limits on focusing of the e-beam.

There are many constraints on the choice of the e/p working point of the HERA collider. Nonlinear resonances for protons, synchro-betatron resonances, spin-orbit resonances for leptons and proton-lepton coupling-resonances need to be carefully avoided to prevent loss of spin polarization and damage to the proton beam, which causes increased backgrounds and loss of luminosity. Therefore the tunes are limited to a narrow window of

$$
\begin{aligned}
& Q_{h o r}^{e}=47.147 \pm 0.002 ; Q_{v e r t}^{e}=47.215 \pm 0.005 \\
& Q_{h o r}^{p}=31.294 \pm 0.002 ; Q_{\text {vert }}^{p}=32.297 \pm 0.002
\end{aligned}
$$

With the tunes inside this window and for present intensities, the lifetime, the emittance growth and the general stability of the proton are excellent in collision. The specific luminosity stays nearly constant over many hours and background conditions are tolerable. The proton beam-beam tuneshift parameters are

$$
\Delta \nu_{x}^{p}=0.0014 ; \Delta \nu_{y}^{p}=0.0007
$$

which are apparently still below the beam-beam limit. At present, the e-beam experiences beam-beam tuneshifts of $\Delta \nu_{x}^{e}=0.0026 ; \Delta \nu_{x}^{e}=0.0032$. From the 1996 experience (with a larger $\beta_{y}^{2}$ value at the IP), the tuneshift limit is expected to occur at values above 0.04 . This means that no substantial increase in luminosity can be achieved by providing a smaller proton emittance.

Increasing the e-beam intensity could provide further increase of luminosity until the yet unexplored limit of the proton beam-beam tune shift is reached. However because of the high costs of $\mathrm{rf}$ power installation and consumption, this would be a very ineffective way to increase luminosity. Increasing the proton beam brightness is also quite expensive because is requires an upgrade of the linear accelerator or another small booster synchrotron or exotic beam cooling techniques. Taking into account that the e-beam beambeam tuneshift does not have a large margin, this way to increase luminosity is not attractive either. The most effective way to increase HERA luminosity by a large factor compared to the design value is therefore to remove the limit on the proton beam $\beta$-functions by a new IR geometry. This will be discussed in the next section.

\section{HERA LUMINOSITY UPGRADE}

HERA beams collide head-on to avoid synchro-betatron resonances and loss of luminosity due to the long proton bunches. The beams need to be separated before the proton beam can be focused by strong low- $\beta$ quadrupoles. In the present lattice this is accomplished by a dipole field generated by displaced e-beam low- $\beta$ quadrupoles and by making use of the different magnetic rigidity of the two beams. A number of constraints limit the $\beta$ in the present lattice. The hard synchrotron radiation generated upstream of the experiment by the separator field is a potential problem for the detector which limits the strength of separating fields. The separator field is a part of the spin rotator. Spin transparency of the beam optics is required. Dispersion generated in the separator gives rise to the excitation of synchrobetatron resonances in the adjacent rf sections. The result is that the first quadrupole magnet which focuses the protons can only be placed $27 \mathrm{~m}$ away from the interaction point and the design values for the $\beta$ function at the IP are rather large with $\beta_{x}^{*}=10 \mathrm{~m}$ and $\beta_{y}^{*}=1 \mathrm{~m}$. Meanwhile these values have been reduced to values of $7 \mathrm{~m}$ and $0.5 \mathrm{~m}$ respectively by using up all the aperture margin in the low $\beta$-quadrupoles.

A new interaction region has been laid out to overcome these limitations. It is based on two concepts: putting separator magnets much closer to the interaction point and making use of new types of septum quadrupoles. Both concepts help to achieve smaller $\beta$-functions at the IP.

The early separation fields will be generated by air-coil magnets inside the colliding beam detector. They are more than twice as strong as the present separators. The intense, hard synchrotron radiation (with a radiation power of $P_{\text {rad }}=25 \mathrm{~kW}$, and a critical energy of $E_{c r i t}=120 \mathrm{keV}$ ) does not hit any components inside the detector and gets absorbed further downstream. The low- $\beta$ magnets of the e-beam which are aligned on the e-beam axis need a gap between the coils to make way for the synchrotron radiation fan. At about $11 \mathrm{~m}$ from the IP, a factor of more than two closer than in the present lattice, the two beams are sufficiently separated by $56 \mathrm{~mm}$. This is enough for a new type of quadrupole septum[11] which focuses only the proton beam. It is described below. The new lattice concept is illustrated in Fig. 2 which shows a top view of the magnets around the IP together with the synchrotron radiation fan.

The new scheme allows the $\beta$-functions of the protons to be reduced by a large factor. In order to fully exploit this advantage, the lepton beam must be focussed more tightly as well. Since large chromatic effects must be avoided, it is essential that the separator magnets have a gradient and provide some focusing to the e-beam. The outer diameter of these magnets is limited to $190 \mathrm{~mm}$ and the magnets have to be very compact. In addition sufficient aperture must be provided to avoid obstruction of the synchrotron radiation fan at the downstream end. This requires a non-conventional coil design. The conceptual design of this magnet promises a gradient of $7.6 \mathrm{~T} / \mathrm{m}$ and an average 


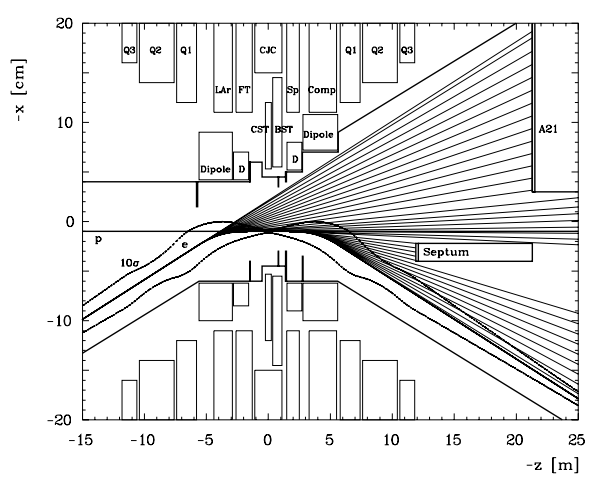

Figure 2: Top View of the New HERA IR: The beams are separated by a relatively strong dipole field generated by air-coil combined function magnets in the colliding beam detector. The intense synchrotron radiation fan passes through gaps between the coils of two iron quadrupoles which form, together with the air-coil magnets, a low- $\beta$ triplet, for the e-beam. At about $11 \mathrm{~m}$ the two beams are sufficiently separated so that the first septum quadrupole to focus the proton beam can be placed there. The thin septum is protected by a small water-cooled absorber. Most of the synchrotron radiation passes along midplane gaps through the septum quadrupole and gets absorbed at $25 \mathrm{~m}$ by a power absorber.

dipole field of $0.22 \mathrm{~T}$. About $100 \mathrm{~kW}$ of electrical power are dissipated in each of the eight separator magnets. These magnets present a challenge for the technical design. Figure 3 shows a cross-section of the separator magnet.

The new type of septum quadrupole has been developed from the half-quadrupole concept used in the present HERA lattice[11]. Since the field at the mirror plate in the magnet midplane is zero, the mirror plate can be reduced to zero thickness at this point. In this way a triangular cut out has been designed which separates the region of zero magnetic field and a strong gradient by a septum of quasi zero thickness. Figure 4 shows a cross-section of the septum quadrupole.

The second part of the first focusing lens of the protons is a current septum quadrupole which resembles the one of the PEPII design [12]. A layout is shown in Fig. 5.

As a result, the $\beta$-functions of the protons at the IP can be reduced from the design values $\beta_{x p}=10 \mathrm{~m}, \beta_{y p}=1 \mathrm{~m}$ to $\beta_{x p}=2.45 \mathrm{~m}, \beta_{y p}=0.18 \mathrm{~m}$. The same aperture of $12.5 \sigma_{x, y}$ is provided, which leaves sufficient margin. The vertical $\beta$ is, with $0.18 \mathrm{~m}$, touching on the bunch length limit $\left(\sigma_{s} \simeq(12-19) \mathrm{cm}\right)$ and cannot be further reduced whereas in the horizontal plane there is still some room for improvement by giving up safety margins (present beam optics for protons in luminosity opertion provides only $10 \sigma_{x, y}$ of aperture).

Using the focusing provided by the separator magnets, the $\beta$-functions of the e-beam must be reduced by a large factor as well. In addition, stronger focusing is required in the arc to produce an emittance as small as $\varepsilon_{e x}=22 \mathrm{~nm}$.

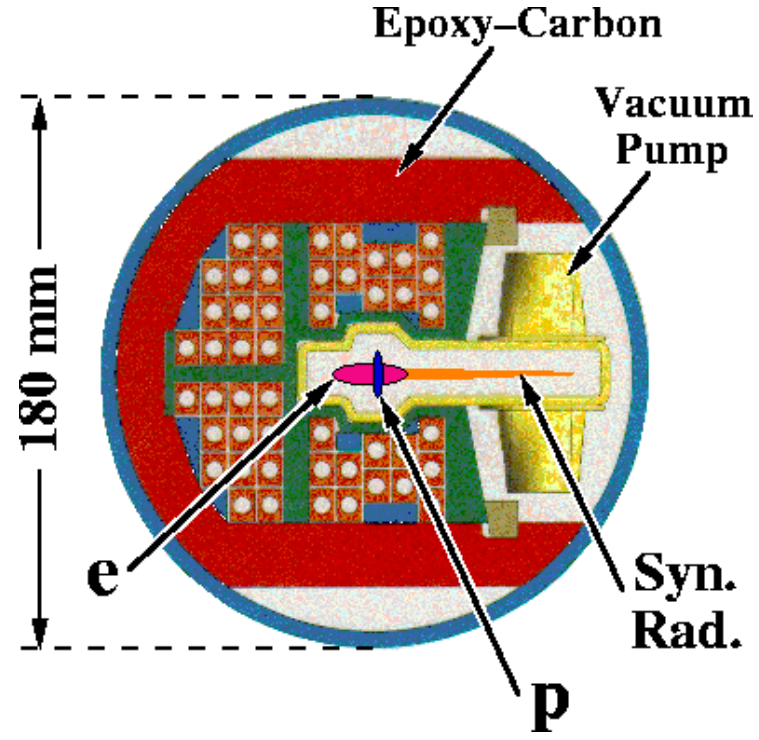

Figure 3: Section of an Air-coil Separator Combined Function Magnet which is placed inside the colliding beam detectors. The magnet fits into a circle of only $180 \mathrm{~mm}$ diameter. It has a length of $2 \mathrm{~m}$. Its average field is about $0.22 \mathrm{~T}$, its gradient is $7.6 \mathrm{~T} / \mathrm{m}$.

This is accomplished with a beam optics with 90 degree $\beta$ tron phase advance in the horizontal plane and 60 degree phase advance in the vertical plane. With this arrangement second order chromatic effects can be kept under control and sufficient dynamic aperture be provided. Table 1 gives the main parameters for the upgraded HERA lattice.

\begin{tabular}{|l|r|}
\hline Lepton Beam Energy & $30 \mathrm{GeV}$ \\
Proton Beam Energy & $820 \mathrm{GeV}$ \\
Colliding Bunches & 174 \\
Leptons per Bunch & $4.18 \cdot 10^{10}$ \\
Protons per Bunch & $10 \cdot 10^{10}$ \\
Lepton Beam Current & $58 \mathrm{~mA}$ \\
Lepton Emittance & $22 \mathrm{~nm}$ \\
Proton Emittance & $5 \mathrm{~nm}$ \\
Lepton Emittance ratio & 0.18 \\
Proton $\beta_{x, y}^{*}$ at IP & $2.45 \mathrm{~m} / 0.18 \mathrm{~m}$ \\
Lepton $\beta_{x, y}^{*}$ at IP & $0.63 \mathrm{~m} / 0.27 \mathrm{~m}$ \\
$\sigma_{x}^{*} \times \sigma_{y}^{*}$ & $118 \mu \mathrm{m} \times 32 \mu \mathrm{m}$ \\
b-b tuneshift e $\Delta \nu_{x, y}^{e}$ & $0.027,0.041$ \\
b-b tuneshift p $\Delta \nu_{x, y}^{p}$ & $0.0017,0.00046$ \\
Specific Luminosity & $1.64 \cdot 10^{30} \mathrm{~cm}^{-2} \mathrm{~s}^{-1} \mathrm{~m}^{-2}$ \\
Luminosity & $7.36 \cdot 10^{31} \mathrm{~cm}^{-2} s^{-1}$ \\
Improvement factor & 4.9 \\
\hline
\end{tabular}

Table 1: Parameters for Upgraded HERA IR Lattice

There are a number of difficulties which associated with this layout. There are many technical subtleties of mutual forces between the detector field and machine fields, with supports inside the detector, with cooling supply inside the detector with high power synchrotron radiation absorbers, with on-line alignment system, with technical layout of the 


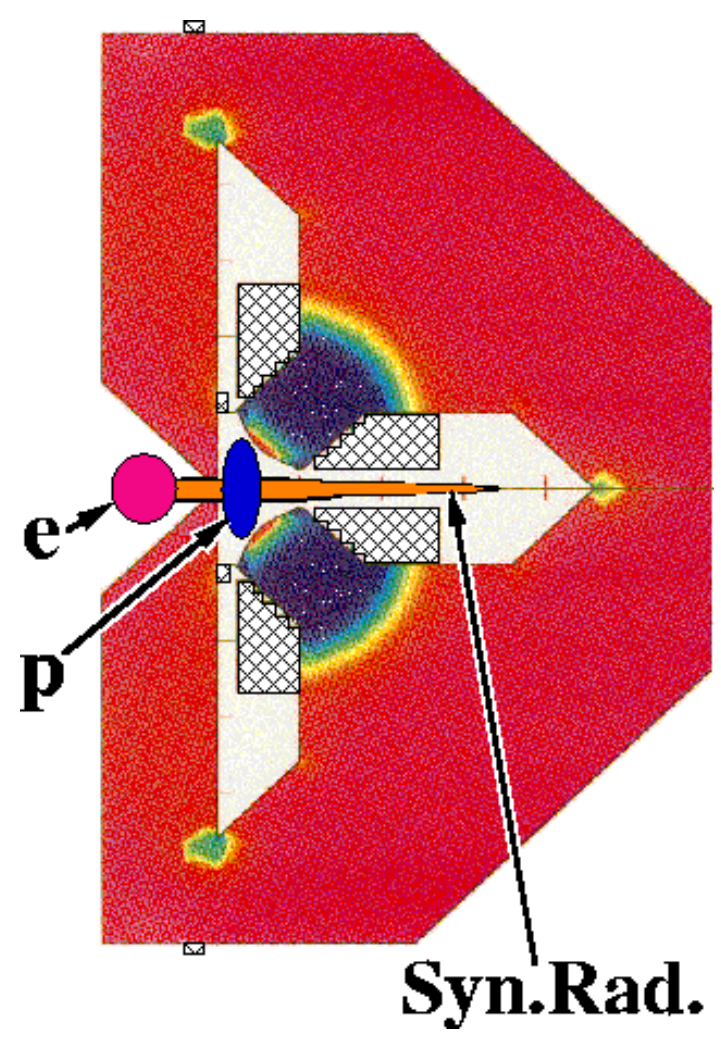

Figure 4: Cross Section of the septum quadrupole, the first focusing element for the protons next to the IP. Note the triangular cut-out which provides the field free space for the e-beam.

septum magnet which are described in a conceptual design report $[14,13]$. Many difficulties with emittance and chromaticity control and dynamic aperture have been resolved. The spin dynamics in the new IR with uncompensated solenoid fields and tilted beam orbits is quite delicate but the problems appear to be solvable.

\section{CONCLUSIONS}

HERA has made good progress in recent years and is about to reach the limit of its design performance. Major technical improvement programmes are underway to overcome the limitations on beam current, overall reliability and the problem with breakdown of the electron beam lifetime.

Further progress in luminosity yield beyond the design values must come from an aggressive new design of the interaction region. A conceptual design of the HERA luminosity upgrade has been made and the feasibility of a luminosity increase by a factor of five compared to the design value has been demonstrated. The project is about to enter a detailed engineering layout. The expectations are to install the new interaction region components in the winter shut-down 1999/2000 providing high lepton-proton luminosity for the next decade.

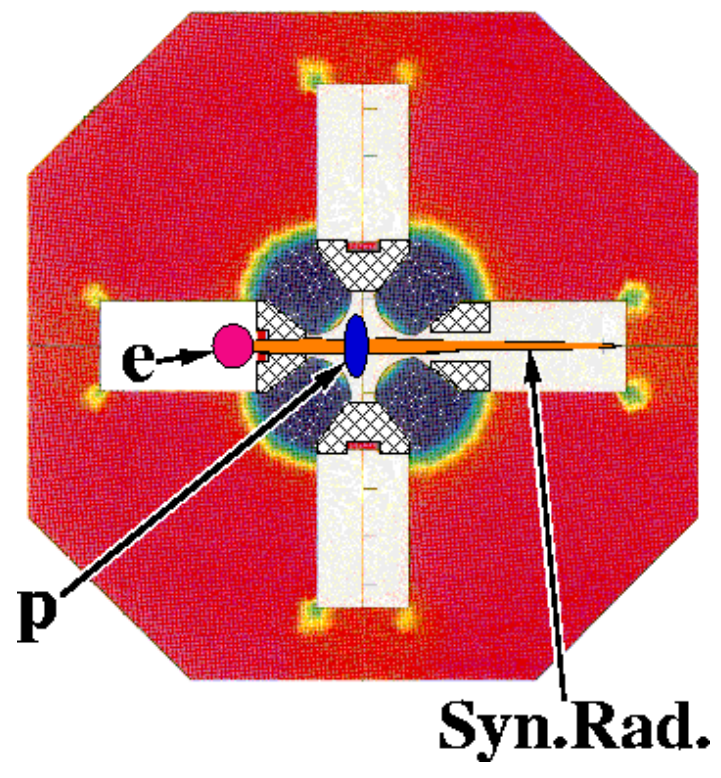

Figure 5: Cross Section of the quadrupole with a septumcoil.

\section{REFERENCES}

[1] "HERA, A Proposal for a Large Electron Proton Colliding Beam Facility at DESY", DESY HERA81-10 1981

[2] R. Bacher, these proceedings

[3] J. Eckolt, these proceedings

[4] W. Bialowons, "Status of HERA" Proceedings of the EPAC96, Sitges (1996) p.3

[5] D. Kelly et al., Proceedings of the PAC95, Dallas (1995)

D.Kelly, W. Bialowons, K.Wittenburg, Proceedings of the EPAC96, Sitges (1996)

[6] D. Kelly, these proceedings

[7] D.P. Barber, "The HERA Polarimeter and the First Observation of Electron Spin Polarisation at HERA", Nuclear Instruments and Methods A329(1993) pp. 79-111;

D. Barber et al., Phys.Letters B343 (1995) pp. 436-443

[8] C. Benvenuti, VACUUM 37 (1980) p. 699

[9] O. Bruening, W.Fischer, F. Schmidt and F. Willeke, DESY HERA 95-05 (1995)

[10] W. Ebeling, J. Maidment, these proceedings

[11] M. Marx, B. Parker and H. Wuempelmann, Proceedings of the EPAC96, Sitges (1996) 2170

[12] PEPII, An Asymmetric B-Factory at SLAC, SLAC 418, LBL-PUB 5379 (1993)

[13] G. Ingelmann, A. De Roeck, R. Klanner, ed's., Procedings of the Workshop on Future Physics of HERA, DESY 19951996 (1996)

[14] U. Schneekloth ed., "Conceptual Design of the HERA Luminosity Upgrade", DESY-HERA, in preparation 